\title{
Growth stress in tungsten carbide-diamond-like carbon coatings
}

\author{
B. R. Pujada, ${ }^{1,2, a)}$ F. D. Tichelaar, ${ }^{3}$ W. M. Arnoldbik, ${ }^{4}$ W. G. Sloof, ${ }^{2}$ and \\ G. C. A. M. Janssen ${ }^{2, b)}$ \\ ${ }_{1}^{1}$ Materials Innovation Institute M2i, Mekelweg 2, 2600 GA Delft, The Netherlands \\ ${ }^{2}$ Department of Materials Science and Engineering, Delft University of Technology, Mekelweg 2, \\ 2628 CD Delft, The Netherlands \\ ${ }^{3}$ Kavli Institute of Nanoscience, National Centre for HTEM, Delft University of Technology, Lorentzweg 1, \\ 2628 CJ Delft, The Netherlands \\ ${ }^{4}$ Department of Physics and Astronomy, Surfaces and Interfaces Devices, Faculty of Science, \\ Utrecht University, P.O. Box 80.000, 3508 TA Utrecht, The Netherlands
}

(Received 13 May 2008; accepted 12 December 2008; published online 2 February 2009)

\begin{abstract}
Growth stress in tungsten carbide-diamond-like carbon coatings, sputter deposited in a reactive argon/acetylene plasma, has been studied as a function of the acetylene partial pressure. Stress and microstructure have been investigated by wafer curvature and transmission electron microscopy (TEM) whereas composition and energy distribution functions of positive ions were obtained by electron probe microanalyzer, elastic recoil detection analysis, and mass-energy analyzer (MEA). It has been observed that the compressive stress decreases with increasing acetylene partial pressure, showing an abrupt change from -5.0 to $-1.6 \mathrm{GPa}$ at an acetylene partial pressure of $0.012 \mathrm{~Pa}$. TEM micrographs show that by increasing the acetylene partial pressure in the plasma from 0 to $0.012 \mathrm{~Pa}$, the microstructure of the coating changes from polycrystalline to amorphous. MEA results show that the most probable energy of positive ions bombarding the substrate during deposition in pure argon and argon/acetylene atmosphere is the same. Based on the results, it is concluded that the huge variation in the compressive stress at low acetylene partial pressures is due to a change in the microstructure of the coating from polycrystalline to amorphous and not to the energy of positive ions bombarding the film. (c) 2009 American Institute of Physics. [DOI: 10.1063/1.3073890]
\end{abstract}

\section{INTRODUCTION}

The understanding and control of the mechanical and tribological properties of tungsten carbide-diamond-like carbon (WC-DLC) coatings and multilayers, prepared by reactive sputter deposition processes, have become subjects of great interest. The low coefficient of friction against steel combined with the high wear resistance and chemical inertness make WC-DLC very attractive for industrial applications, such as protective coatings. ${ }^{1-7}$ Thin films on a substrate are usually in a stressed state. The stress in the film can conveniently be described as the sum of a thermal stress and an intrinsic stress. The thermal stress is due to a different thermal expansion of substrate and film while cooling from deposition temperature to room temperature. The intrinsic stress is due to the growth process itself. The intrinsic stress can either be tensile or compressive. ${ }^{8-10}$ The control of the stress in thin films is of crucial interest because high intrinsic stress values can cause mechanical failure, such as delamination or cracking of the film, limiting therefore the applicability of the coating.

Previous investigations on WC-DLC coatings deposited by reactive rf-magnetron sputter deposition in an argon/ hydrocarbon atmosphere have shown dependence of the film density, hardness, coefficient of friction, and stress on the

\footnotetext{
${ }^{a)}$ Present address: Brazilian Center for Physics Research (CBPF), Rio de Janeiro, Brazil. Electronic mail: brpujada@cbpf.br.

b) Author to whom correspondence should be addressed. Electronic mail: g.c.a.m.janssen@tudelft.nl.
}

substrate bias and the amount of the hydrocarbon gas in the plasma. ${ }^{1-4}$ Recently, we reported that by increasing the acetylene gas flow in the argon/acetylene plasma, the hardness and compressive stress decrease. ${ }^{1} \mathrm{X}$-ray diffraction analysis shows that films deposited in a pure argon atmosphere, consist of polycrystalline WC grains, whereas those deposited in argon/acetylene atmosphere are amorphous. In the present paper, we investigate the influence of the composition, microstructure, and energy of the positive ions bombarding the film on the intrinsic stress.

\section{EXPERIMENTAL}

WC-DLC coatings have been deposited by reactive $\mathrm{rf}$ unbalanced magnetron sputter deposition from a WC target in an argon/acetylene atmosphere onto $100 \mathrm{~mm} \mathrm{Si}$ wafers with (001) orientation. The thickness of the $\mathrm{Si}$ wafer is $525 \mu \mathrm{m}$. Coating thicknesses were measured by crosssectional scanning electron microscopy micrographs (not shown here). It varies from 255 to $1100 \mathrm{~nm}$ when increasing the acetylene partial pressure from 0 to $0.075 \mathrm{~Pa}$. The experimental details of the film sputter deposition process have been reported previously. ${ }^{1}$ The acetylene partial pressure was measured using a mass-energy analyzer. The internal stress of the WC-DLC coatings was calculated from the measured curvature of the substrate wafer before and after deposition and by using the Stoney equation for stress measurements. ${ }^{8}$

The tungsten and carbon content in the WC-DLC coatings was measured by electron probe $\mathrm{x}$-ray microanalysis (EPMA). The measurements were carried out with a JEOL 


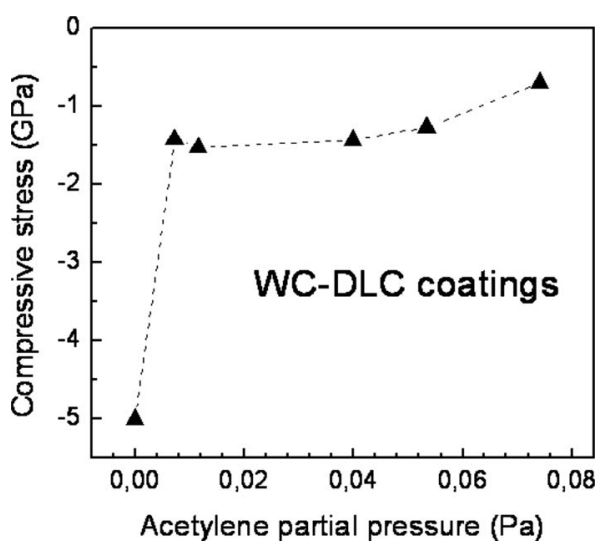

FIG. 1. Compressive stress of the WC-DLC coatings deposited in argon/ acetylene atmosphere as a function of the acetylene partial pressure.

JXA 8900R microprobe using a focused electron beam with energy of $10 \mathrm{keV}$ and a current of $200 \mathrm{nA}$. Prior to each measurement, the surface of the specimen was decontaminated with an in situ air jet for $30 \mathrm{~s}$. The composition at each analysis location of the sample was determined using the

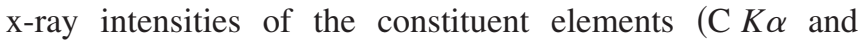
W $M \alpha$ ) after background correction relative to the corresponding intensities of reference materials (i.e., $\mathrm{Fe}_{3} \mathrm{C}$ for carbon and pure $\mathrm{W}$ for tungsten). ${ }^{11}$ The obtained intensity ratios were processed with a matrix correction program based on the $\Phi(p 2)$ method. $^{12}$ In the EPMA, neither hydrogen nor argon were taken into account in the calculation of the tungsten and carbon concentration.

Hydrogen incorporation is an important factor influencing the properties of DLC coatings. ${ }^{13}$ Hydrogen content in the WC-DLC coatings was measured by using elastic recoil detection analysis (ERDA) applying a 6 MV Tandem van der Graaf accelerator. ${ }^{14}$ The detector is a silicon surface barrier and the angle of incidence with the sample surface is $20^{\circ}$. The particles are recoiled from the surface by a $20 \mathrm{MeV} \mathrm{Si}^{4+}$ ion beam. In front of the detector, a 13.2 $\mu \mathrm{m}$ Mylar foil is placed to stop all particles except hydrogen. The recoil angle is $30^{\circ}$.

The microstructure of WC-DLC coatings has been analyzed by cross-sectional transmission electron microscopy (TEM). Microstructural analysis has been performed on a CM30T Philips TEM operating at $300 \mathrm{kV}$. A mass-energy analyzer (MEA), model PPM 442, Pfeiffer Vacuum, was employed for the ion energy distribution measurements. The measurements were carried out in a DN160/100CF reducing cross with the DN160CF flanges along the magnetron axis. A detailed description of the MEA measurements is provided in Refs. 15-17.

\section{RESULTS AND DISCUSSION}

The stress results presented in Fig. 1 are the sum of the intrinsic stress and the thermal stress. However, the thermal stress is small compared to the intrinsic stress. The thermal expansion coefficients of $\mathrm{WC}$ and $\mathrm{Si}$ are almost equal. We estimate a maximum thermal stress for our measurements in the order of $100 \mathrm{MPa}$. Compared to measured stresses in the $\mathrm{GPa}$ range, we neglect the thermal stress. Compressive stress

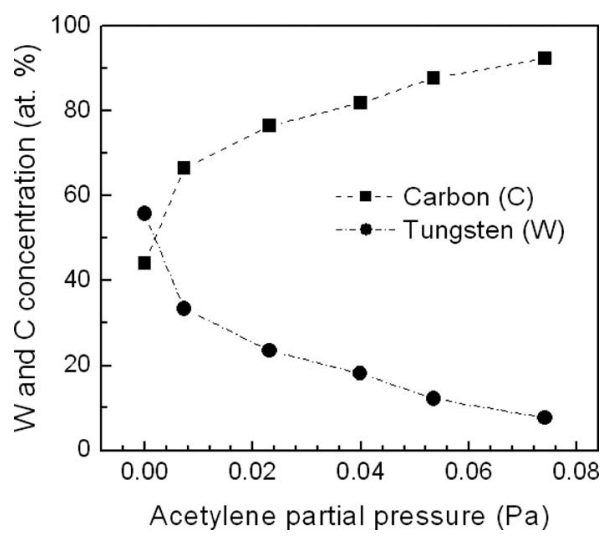

FIG. 2. Carbon (c) and tungsten (w) concentrations in at. $\%$ measured by EPMA, as a function of the acetylene partial pressure.

of the WC-DLC coatings as a function of the acetylene partial pressure from 0 to $0.08 \mathrm{~Pa}$ is presented in Fig. 1. The uncertainty in acetylene partial pressure increases at pressures lower than $0.01 \mathrm{~Pa}$. It is clear that the compressive stress decreases with the acetylene partial pressure. Of particular interest is that as soon as the depositions are carried out with addition of $0.012 \mathrm{~Pa}$ of acetylene partial pressure, the compressive stress abruptly decreases from -5.0 to -1.6 $\mathrm{GPa}$, while further increase of the acetylene partial pressure only results in a moderately lower compressive stress values. The question now is: What causes this huge change in compressive stress? Is it due to a massive change in the ion bombardment? Does the acetylene dramatically change the $\mathrm{Ar}^{+}$density? Or does the acetylene lead to an incorporation of carbon in the growing film, changing the microstructure, and thereby changing the stress?

Figure 2 shows the tungsten and carbon concentration in at. \% determined by EPMA in WC-DLC coatings as a function of the acetylene partial pressure. From Fig. 2, it is evident that when the acetylene partial pressure increases from 0 to $0.08 \mathrm{~Pa}$ in the plasma, the carbon concentration in the film increases monotonically from 44 to 92 at. \% whereas the tungsten concentration decreases from 56 to 8 at. \%. The increase in carbon concentration may be due to the increase of the decomposition rate of the hydrocarbon gas in the plasma with increasing the acetylene partial pressure. The decrease of the tungsten in concentration and also in absolute sense (not shown here) can be associated with a lower sputtering yield of a contaminated WC target. ${ }^{3,4}$

Figure 3 shows the hydrogen concentration depth profiles extracted from ERDA experiments. The depth scale is expressed in atoms $/ \mathrm{cm}^{2}$ of $\mathrm{W}, \mathrm{C}$, and $\mathrm{H}$. The profiles show a hydrogen concentration gradient increasing with depth roughly from 0.1 to 0.8 at. \% in a pure WC layer. For WCDLC coatings deposited at 0.012 and $0.04 \mathrm{~Pa}$ of acetylene gas partial pressure, the hydrogen concentration increases from 3.5 to 4.5 at. $\%$ and 10 to 12 at. \%, respectively. Hence, as expected, the hydrogen content in the WC-DLC coating increases with the acetylene gas partial pressure during deposition.

Figure 4 shows a dark field TEM image and selected area diffraction patterns (SADP) of coatings deposited in a pure argon atmosphere [Fig. 4(a)] and in argon/acetylene at- 


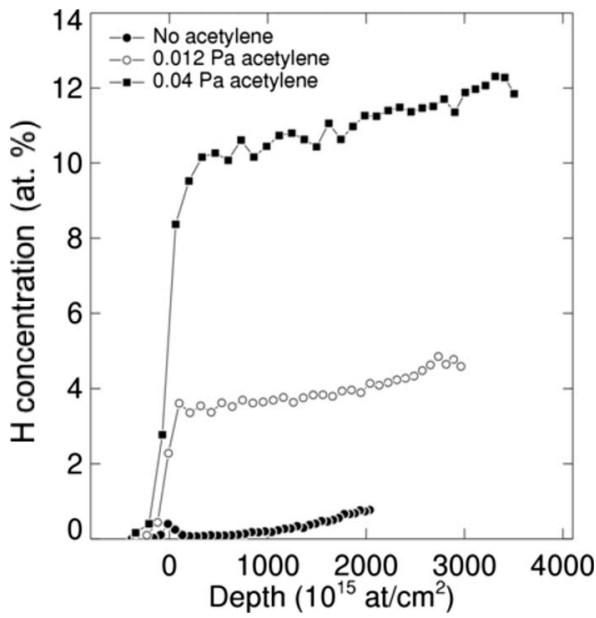

FIG. 3. Hydrogen concentration depth profiles extracted from ERDA experiments of the WC-DLC coatings deposited in pure argon atmosphere and at 0.012 and $0.04 \mathrm{~Pa}$ of acetylene partial pressure. The ends of the curves mark the probing depth of ERDA, which depends on the composition of the sample.

mosphere at $0.012 \mathrm{~Pa}$ of acetylene partial pressure [Fig. 4(b)]. The cross-sectional TEM micrograph of the coating deposited in a pure argon atmosphere exhibits a columnar grain structure parallel to the growth direction with a column width between 10 and $40 \mathrm{~nm}$. The presence of dotted diffraction rings as observed in the SADP image in Fig. 4(a) (inset) indicates a WC polycrystalline phase as previously deduced from XRD. ${ }^{1}$ The tungsten and carbon concentrations were 56 and 44 at. \%, respectively. On the other hand, the crosssectional TEM micrograph and the diffuse diffraction rings

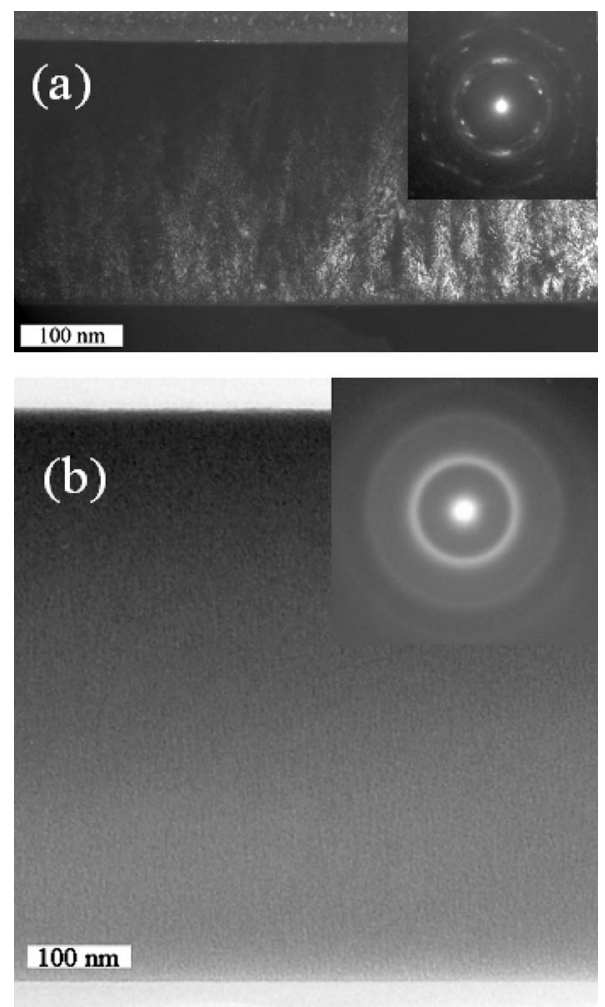

FIG. 4. Cross-sectional TEM micrographs and selected area diffraction patterns of the WC-DLC coatings deposited in a pure argon atmosphere (a), dark field, and $0.012 \mathrm{~Pa}$ of acetylene partial pressure (b), bright field.
TABLE I. $d$ spacings of WC polycrystalline layer obtained by HREM and SADP analysis.

\begin{tabular}{|c|c|c|c|}
\hline \multirow{3}{*}{$\begin{array}{l}\text { Coating } \\
\text { WC } \\
\text { polycrystalline }\end{array}$} & \multicolumn{2}{|c|}{ Technique } & $d$ spacings \\
\hline & HREM & \pm 0.05 & $2.42,2.27,2.26,2.19$ \\
\hline & SADP & \pm 0.03 & $2.48,2.35,2.29,2.20,2.11,1.53,1.30$ \\
\hline
\end{tabular}

present in the SADP image of the WC-DLC coating deposited at $0.012 \mathrm{~Pa}$ of acetylene partial pressure indicate that the coating is amorphous. As indicated in Fig. 1, at the same pressure where the stress drops the microstructure changes from polycrystalline to amorphous.

Table I presents the interplanar $d$ spacings for WC polycrystalline film measured from high-resolution lattice images at different locations in the images, and from SADP using the middle of the band forming such rings. The results for the interplanar $d$ spacing for WC polycrystalline layer are in agreement with previous reports indicating the presence of several phases. $6,18,19$

Figure 5 shows the energy distribution functions of positive ions measured in pure argon plasma and argon/acetylene plasma at an acetylene partial pressure of $0.019 \mathrm{~Pa}$. From the analysis of the MEA measurements, energy profiles were determined for $\mathrm{Ar}^{+}, \mathrm{H}_{2}^{+}, \mathrm{Ar}^{2+}$, and $\mathrm{C}_{2} \mathrm{H}_{2}^{+}$ions. The most probable energy of the ions determined by a plasma potential of $24 \mathrm{~V}$ were found to be independent of the plasma composition. These results indicate no change of the energy of the positive ions bombarding the film during growth under dif-
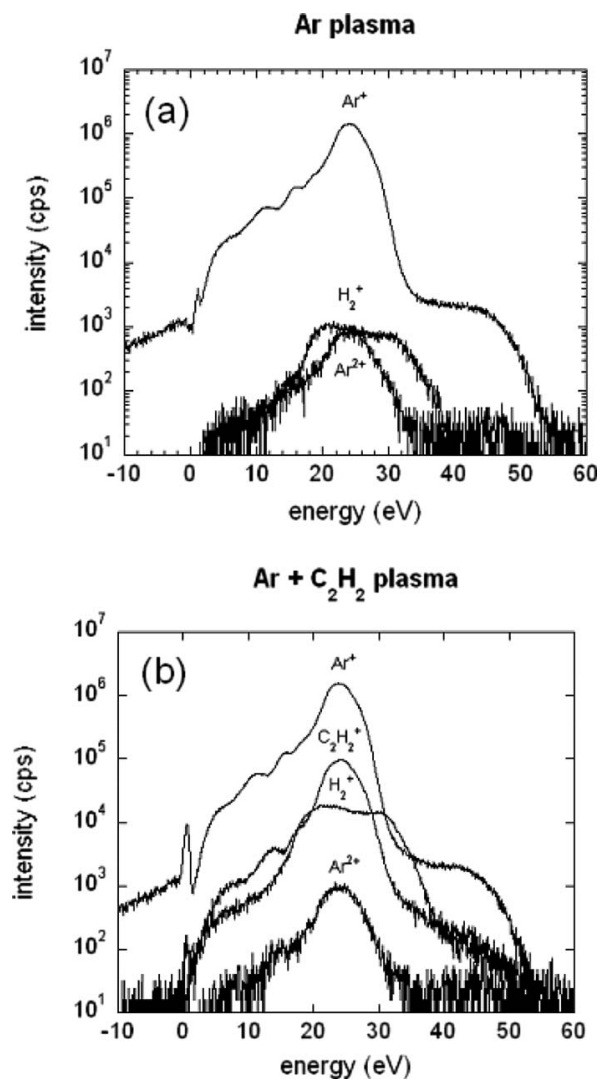

FIG. 5. Energy distribution as a function of positive ions type in pure argon (a) and argon/acetylene (b) plasma measured by MEA. 
ferent atmospheres. In addition, the $\mathrm{Ar}^{+}$flux was maintained constant, while the $\mathrm{C}_{2} \mathrm{H}_{2}^{+}$flux increases with acetylene partial pressure.

WC thin films with high tungsten concentration exhibit a high compressive stress. This high value for compressive stress is probably a consequence of its polycrystalline structure and the high density of pure WC films. By the addition of $0.012 \mathrm{~Pa}$ of acetylene partial pressure in the plasma, the microstructure changes from polycrystalline to amorphous WC as confirmed by TEM analyses resulting in an abrupt decrease of the compressive stress. This change in stress can be due to the change in structure of the film from polycrystalline to amorphous $\mathrm{WC}$, reducing therefore the stress of the film. ERDA shows a continuous increase in hydrogen content with acetylene gas partial pressure. However, the stress in the coatings drops abruptly with increasing the acetylene partial pressure from 0 to $0.012 \mathrm{~Pa}$. The formation of amorphous thin films can be caused by incorporation of a certain amount of hydrogen. From the qualitatively different dependence for acetylene partial pressures of $0.012 \mathrm{~Pa}$ and higher of hydrogen content and stress, we conclude that the variation in intrinsic stress does not depend on the relative amount of hydrogen content in WC-DLC coatings. No change in the positive ion's most probable energy, as shown in Fig. 5, suggest rather limited influence of the positive ions on the microstructure and stress development in the WC-DLC coating. Therefore, these results clearly demonstrate that the abrupt drop in the compressive stress in WC-DLC coatings is due a change in microstructure caused by the chemistry of the growing film. From the tungsten carbon phase diagram, ${ }^{20}$ it can be seen that there is no solubility of carbon in WC. Therefore excess carbon from the acetylene on the surface of the growing film will frustrate the growth of a polycrystalline WC film and give rise to an amorphous film consisting of carbon and tungsten with a carbon to tungsten atomic ratio greater than 1 .

\section{CONCLUSION}

We have presented an experimental study of the influence of the acetylene partial pressure on the compressive stress in WC-DLC coatings. We have found that by increasing the acetylene partial pressure, the carbon and hydrogen concentrations in the coatings increase, resulting in the formation of an amorphous structure as observed in the SADP images. The intrinsic compressive stress decreases to lower values with increasing the acetylene pressure in the plasma. For acetylene partial pressures of $0.012 \mathrm{~Pa}$ and higher, the dependence of the intrinsic stress on the acetylene gas partial pressure does not seem to be related to the amount of the hydrogen incorporated into the WC-DLC coating. We have observed by TEM analysis a difference in the microstructure in WC-DLC coatings deposited in pure argon and argon/ acetylene atmospheres. Since the introduction of acetylene gas does not result in a significant change in the most probable energy of the bombarding positive ions during growth, the contribution of the energetic ion bombardment to the compressive stress is not significant. In conclusion, the abrupt drop in the compressive stress at $0.012 \mathrm{~Pa}$ of acetylene partial pressure is explained by a change in the microstructure from a WC polycrystalline to amorphous.

\section{ACKNOWLEDGMENTS}

This research was carried out under Project No. MC7.02142B within the framework of the strategic Research program of the Materials innovation institute M2i. The authors would like to thank Stanislav Mráz and Jochen M. Schneider (Materials Chemistry, RWTH Aachen University, Germany) for MEA measurements and analysis.

${ }^{1}$ B. R. Pujada and G. C. A. M. Janssen, Surf. Coat. Technol. 201, 4284 (2006).

${ }^{2}$ C. Strondl, N. M. Carvalho, J. Th. M. De Hosson, and G. J. van der Kolk, Surf. Coat. Technol. 162, 288 (2003).

${ }^{3}$ A. Czyzniewski and W. Precht, J. Mater. Process. Technol. 157-158, 274 (2004).

${ }^{4}$ M. Wang, K. Schmidt, K. Reichelt, X. Jiang, H. Hübsch, and H. Dimigen, J. Mater. Res. 7, 1465 (1992).

${ }^{5} \mathrm{Ph}$. Gouy-Pailler and Y. Pauleau, J. Vac. Sci. Technol. A 11, 96 (1993).

${ }^{6}$ C. Rincon, J. Romero, J. Esteve, E. Martinez, and A. Lousa, Surf. Coat. Technol. 163-164, 386 (2003).

${ }^{7}$ B. R. Pujada, F. D. Tichelaar, and G. C. A. M. Janssen, Appl. Phys. Lett. 90, 021913 (2007).

${ }^{8}$ G. C. A. M. Janssen, Thin Solid Films 515, 6654 (2007).

${ }^{9}$ C. A. Davis, Thin Solid Films 226, 30 (1993).

${ }^{10}$ M. M. M. Bilek and D. R. McKenzie, Surf. Coat. Technol. 200, 4345 (2006).

${ }^{11}$ S. Saunders, P. Karduck, and W. G. Sloof, Microchim. Acta 145, 209 (2004).

${ }^{12}$ G. F. Bastin and H. J. M. Heijligers, Scanning 12, 225 (1990).

${ }^{13}$ J. Robertson, Mater. Sci. Eng. R. 37, 129 (2002).

${ }^{14}$ W. M. A. Bik and F. H. P. M. Habraken, Rep. Prog. Phys. 56, 859 (1993).

${ }^{15}$ S. Mráz and J. M. Schneider, Plasma Chem. Plasma Process. 26, 197 (2006).

${ }^{16}$ S. Mráz and J. M. Schneider, J. Appl. Phys. 100, 023503 (2006).

${ }^{17}$ S. Mráz and J. M. Schneider, Appl. Phys. Lett. 89, 051502 (2006).

${ }^{18}$ P. K. Srivastava, T. V. Rao, V. D. Vankar, and K. L. Chopra, J. Vac. Sci. Technol. A 2, 1261 (1984).

${ }^{19}$ A. A. Voevodin, J. P. O’Neil, S. V. Prasad, and J. S. Zabinski, J. Vac. Sci. Technol. A 17, 986 (1999).

${ }^{20}$ Th. B. Massalski, Binary Alloy Phase Diagrams (ASM International, Materials Park, 1990). 